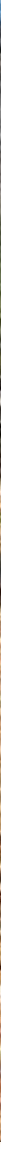

\title{
Anmerkungen zur älteren Geschichte von Geringswalde
}

\author{
Wolfgang Schwabenicky
}

„Im Fürstenwalde bei Geringswalde, einem zu dem Amte Colditz gehörigen Forste, befinden sich noch erkennbare Reste einer Burg, über deren Besitzer und Schicksale auffallend wenige oder eigentlich gar keine Nachrichten vorhanden sind." So schreibt Johannes Falke 1867 in den Mitteilungen des Königlich Sächsischen Vereins für die Erforschung und Erhaltung vaterländischer Geschichts und Kunstdenkmale. ${ }^{1}$ Bei diesen Resten einer Burg handelt es sich um eine große dreiteilige Anlage auf einem nach drei Seiten steil abfallenden Sporn über dem Auenbach etwa zwei Kilometer nordwestlich von Geringswalde. Seit diese Burg wieder in das Bewusstsein der Menschen gerückt ist, beschäftigte man sich damit, ob sie mit dem „fundus castri destructi“ in der Gründungsurkunde des Klosters Geringswalde 1233 in einem Zusammenhang steht, wobei es zu unterschiedlichsten, zum Teil sich widersprechenden Angaben und Schlussfolgerungen kam. Eine erste Einordnung versuchte 1777 Gottfried August Bernhardi. Nach seinem Ermessen konnte es sich nicht um eine Burg derer von Schönburg, Eigentümer von Geringswalde, gehandelt haben, da sie im Fürstenwald liegt, welcher schon seit langer Zeit Eigentum der Markgrafen von Meißen war und in das Amt Colditz gehörte. ${ }^{2}$ Eine längere Abhandlung widmete der Burg 1843 der Advokat Karl Gautsch. ${ }^{3}$ Er schilderte aufgrund von Akten ausführlich die Schatzgräberei in der $\mathrm{Ru}-$ ine auf dem Hauskellerberg, beschrieb einige Funde und bildete einen Plan von 1738 ab. Er vermutete, wie auch schon Bernhardi, die zerstörte Burg am unteren Ende von Geringswalde, hielt aber für möglich, dass die Anlage im Fürstenwald der Sitz eines schönburgischen Vasallen war oder dass sie gar zur Herrschaft Colditz gehörte. Auch für Johannes Falke galt als sicher, dass die zerstörte Burg von 1233 nicht die Burg im Fürstenwald war, sondern ein Schloss auf dem sogenannten Burgberg im Westen der Stadt Geringswalde. ${ }^{4}$ Er schloss sich damit sowohl Gottfried August Bernhardi als auch Anton Tobias an. ${ }^{5}$ Reinhold Hofmann ließ es offen, ob die Burg auf dem Hauskellerberg Rochlinti war und im Besitze der Schönburger war. ${ }^{6}$ Gerhard Billig und Heinz Müller ${ }^{7}$ folgten der lokal-
Geringswalde, Altes Schloss. Sporn, auf dem die Kernburg stand. Foto: Klaus Ebert

Der Beitrag ist Klaus Gumnior dem langjährigen Herausgeber der "Sächischen Heimatblätter" zum 75. Geburtstag gewidmet. 
Geringswalde, Altes Schloss, Graben an der Nordseite Foto: Klaus Ebert

4 Falke 1867(wie Anm. 1), S. 22 f.

Tobias, Anton: Regesten des Hauses Schönburg vom urkundlichen Auftreten desselben bis zum Jahre 1326. Zittau 1865 , S. 12.

6 Hofmann, Reinhold/Müller, Conrad: Gründung und Geschichte des Klosters Geringswalde. Aus dem Nachlasse des Studienrates Professors Dr. Reinhold Hofmann erweitert und herausgegeben von Dr. Conrad Müller. Waldenburg 1920, S. $36 \mathrm{f}$.

Billig, Gerhard/Müller, Heinz: Burgen Zeugen sächsischer Geschichte, Neustadt a. d. Aisch 1998, S. 96 f.

Durch diese Formulierung wird suggeriert, dass die beiden „sicheren schönburgischen Wehranlagen“ schriftlich überliefert sind, was nicht stimmt. Außerdem befindet sich die dritte Burg nicht nordwestlich der Rinnmühle, sondern ca. einen Kilometer südsüdöstlich der Rinnmühle. Mit den beiden „sicheren“ Wehranlagen ist einmal der Buchberg ca. 200 m südlich des Standortes des Klosters und zum anderen die Halbige (Burgberg), ein am südwestlichen Ende der Stadt auf einem Sporn oberhalb der Dammmühle liegenden Platz, der von Häusern umbaut ist. An keinen der beiden Örtlichkeiten sind Anzeichen einer ehemaligen Befestigung zu finden. Im Dezember 1994 wurde auf dem Sporn an der Stelle, wo ein Abschnittsgraben zu erwarten wäre, aufgrund einer geplanten Baumaßnahme ein Baggerschnitt gezogen, der zeigte, dass hier in ca. $0,5 \mathrm{~m}$ Tiefe der ungestörte Felsen liegt.

Sieber, J. G.: Beiträge zur Geschichte der Stadt Hartha. In: Hermann Gläsers Harthaer Heimatbuch. Bd. . Hartha 1929, S. 1-168, hier S. $20 \mathrm{f}$

10 Urkunde vom 2. Januar 1233 über die Stiftung des Klosters Geringswalde in Sächsisches Staatsarchiv, Staatsarchiv Chemnitz, 30570 Urkunden der Linie Hinterglauchau. Sie wurde wohl erstmals gedruckt bei Weller, Johann Gottfried: Altes aus allen Theilen der Geschichte. Chemnitz 1762, S. 573575. Von hier ist die Urkunde wahrscheinlich von Bernhardi 1777 (wie Anm. 2), S 53 f. übernommen worden. Beide Urkundentexte sind fehlerhaft. Tobias 1865 (wie Anm. 5), bringt S. 10-12 den Text der Urkunde zusammen mit einem längeren Kommentar. Als Faksimile befindet sich die Urkunde als Beilage bei Posse, Otto: Die Urahnen des Fürstlichen und Gräflichen Hauses Schönburg, Dresden 1914. Danach hat Conrad Müller die Edition und Übersetzung besorgt (Hofmann/Müller 1920 [wie Anm. 6], S. 26-30), deren wir uns in diesem Aufsatz bedienen.

11 Diese drei Orte liegen abseits vom Geringswalder Gebiet. Sie bleiben bei unseren Ausführungen außer Betracht.

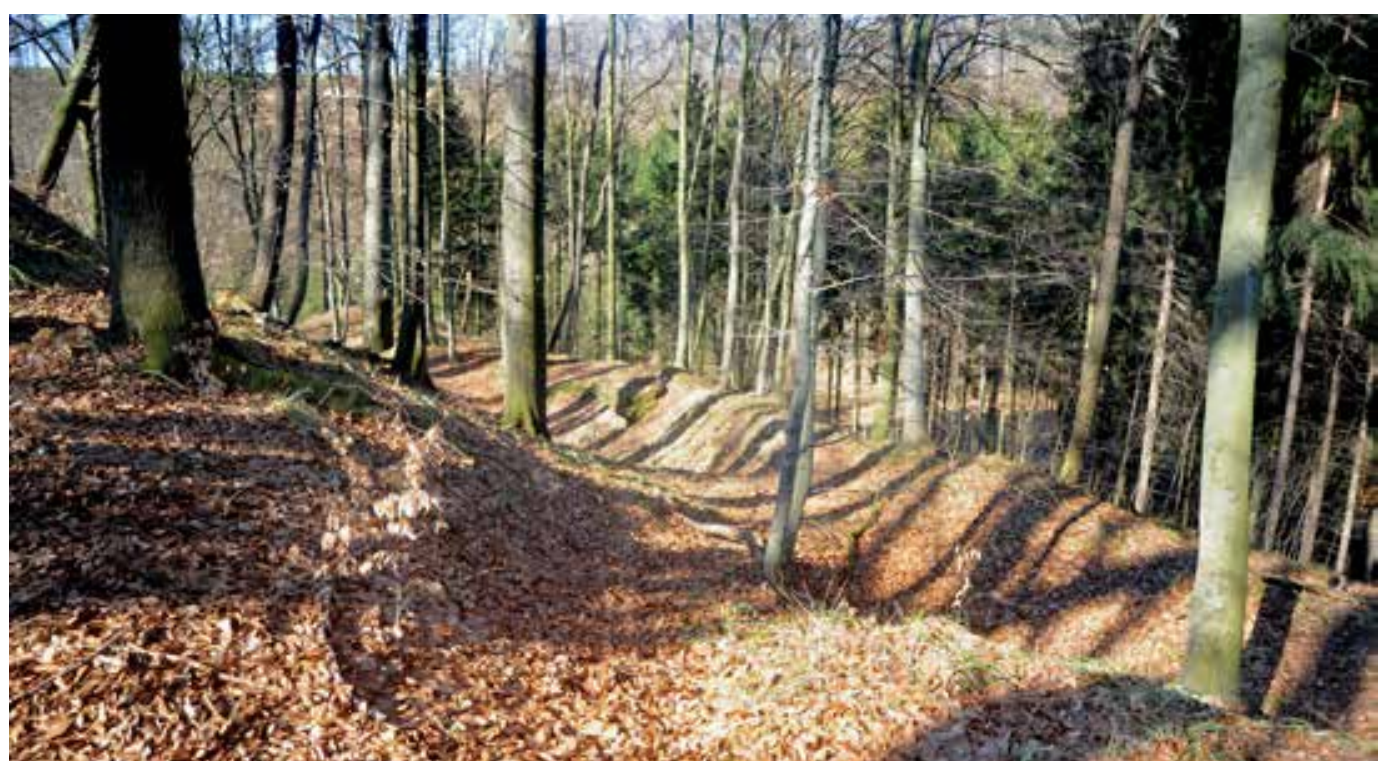

geschichtlichen Tradition, indem sie schrieben: „Neben den beiden sicheren schönburgischen Wehranlagen in Kloster und Stadt Geringswalde steht eine dritte stattliche dreikernige Burg ohne jegliche schriftliche Erwähnung im Wald nordwestlich der Rinnmühle."8 Bereits für J. G. Sieber stand entgegen anderer Annahmen fest, dass die Burg im Fürstenwald der Sitz derer von Schönburg vor 1233 gewesen war. ${ }^{9}$ Es scheint also gerechtfertigt, die Burg und die ältere Geschichte des Geringswalder Ländchens noch einmal näher zu beleuchten.

Ausgangspunkt zur Betrachtung der Geschichte der Landschaft ist meist die Urkunde über die Gründung des Benediktinerklosters Geringswalde aus dem Jahre $1233 .{ }^{10}$ Aus dieser am 2. Januar des Jahres 1233 durch Hermann von Schönburg ausgestellten Urkunde erfahren wir, dass dieser ein Jungfrauenkloster in Geringswalde ausgestattet und erbaut hat. Das Kloster erhielt als Ausstattung die Patronatsrechte über die Kirchen in Weistropp und Leubnitz sowie sechs Hufen in Zschannewitz, ${ }^{11}$ des weiteren 16 Hufen in „antiquo Gerungeswalde“ (Altgeringswalde), drei Mühlen mit allen Zubehör, fünf Hofstätten sowie den Grund und Boden der zerstörten Burg und des verwüsteten Städtleins ${ }^{12}$ mit dem anliegenden Fischteiche (,V areas et fundum castri destructi et oppidi desolati cum piscina eciam adiacente“), außerdem den Wald nahe Hoyersdorf mit dem Dorf selbst. Außerdem gestattete er, dass, wenn Weideplätze in seinem Forst geschaffen werden, sich das Kloster ein Drittel davon nehmen kann, und dass das Kloster zur Errichtung der Gebäude das Bauholz im Wald schlagen kann.

Das Gebiet um Geringswalde gehört nach Ernst Neef $^{13}$ geographisch zum Erzgebirgsvorland, und zwar in dessen als Mittelsächsisches Löß- lehmgebiet bezeichneten nordöstlichen Teil. Die Rumpfflächen sind mit Lößlehm bedeckt, der weit günstigere Voraussetzungen für landwirtschaftliche Nutzung mit sich bringt, als dies bei den Verwitterungsböden des Erzgebirges der Fall ist. Allerdings wird die Fruchtbarkeit der Lößgebiete weiter nördlich bei weitem nicht erreicht. In den Gebieten des Altsiedellandes finden sich außerordentlich günstige Böden und ein relativ mildes Klima. Das Erzgebirgsvorland war vor der Mitte des 12. Jahrhunderts mit Ausnahme der engeren Landschaft um Rochlitz unbesiedelt. Seit dem 12. Jahrhundert musste aufgrund der Vermehrung der Bevölkerung auch auf weniger günstige $\mathrm{Ge}$ genden ausgegriffen werden, so auch auf das Erzgebirgsvorland und das Erzgebirge. Die Ausgangspunkte für die Besiedlung unserer Gegend waren die Zentren des Altsiedellandes. Die Verfügungsgewalt über die Wildlandgrenze ermöglichte die Rodung der Wälder und die Bildung neuer Herrschaften. ${ }^{14}$ Für unser Gebiet war das Rochlitz, Colditz und Leisnig. Colditz und Leisnig war seit 1158 Reichsgut und stand unter unmittelbarer Verfügungsgewalt des Kaisers ${ }^{15}$, der somit auch über das angrenzende Wildland (Wald) entschied. Die Rodung und Besiedlung der Wälder wurde unter der Führung des Reichsadels durchgeführt. Sie erhielten ein Stück Wildland, das nicht immer am Rand des Altsiedellandes liegen musste, zu Lehen oder auch als Eigen. So begann beispielsweise der Rodungsstreifen der Herren von Schellenberg am Frauenholz südlich von Frankenberg und zog sich entlang der Zschopau aufwärts. ${ }^{16}$

Einen ähnlichen Waldstreifen erhielten die aus der Naumburger Gegend stammenden vom Herrenstand zur Reichsministerialität übergetretenen Herren von Schönburg17 vermutlich 
schon vor 1150, spätestens aber von Friedrich Barbarossa (um 1122-1190) als Eigen. Dieser Streifen erstreckt sich in Südwest-Nordost-Richtung etwa parallel zur Wildlandgrenze bei Leisnig. Er wird berührt von einem von Leisnig ausgehenden Zweig des Alten böhmischen Steiges, der 1185 in der Grenzbeschreibung des Klosters Altzelle als Westgrenze des klösterlichen Gebietes mit „ad cumulum in Bukendorf ab illo per antiquam Boemie semitam“ erwähnt wird ${ }^{18}$ und der bei Waldheim die Zschopau querte. Die von den Schönburgern gegründeten Orte lassen sich mit einiger Sicherheit aus der urkundlichen Überlieferung erschließen. Auf alle Fälle gehören dazu die Dörfer, über die das Kloster Geringswalde die Obergerichte besaß, welche es 1261 von Friedrich von Schönburg im schönburgischen Gerichtsbezirk und Land bei der Stadt Geringswalde (,in nostra jurisdictione et territorio juxta opidum Gerungiswalde“) erhalten hatte. ${ }^{19}$ Nach dem Erbzinsregister von $1590^{20}$ sind das folgende Orte: Hermsdorf, Altgeringswalde, Aitzendorf, Dittmanndorf, Hoyersdorf, Hilmsdorf, Langenau, Schönerstädt, Flemmingen und Aschershain. Altgeringswalde und Hoyersdorf werden bereits in der Gründungsurkunde 1233 genannt. Das Pfarrdorf Schönerstädt erwarb 1271 der Geringswalder Probst Walter, wann und von wem ist nicht bekannt, und schenkte es dem Kloster. ${ }^{21}$ Es scheint wohl vorher Heinrich von Polek gehört zu haben. Dieser hatte vor 1245 bereits das zum Pfarrsprengel Schönerstädt gehörige Langenau und die Dörfer Gersdorf und Kieselbach an das Kloster Buch verkauft. ${ }^{22}$ Heinrich von Polek (Polkenberg), der schon in der Stiftungsurkunde des Klosters Geringswalde als Zeuge auftritt, wird von Walter Schlesinger als ein Lehnsmann der Schönburger angesehen. ${ }^{23}$ Langenau ist später, wann genau ist unbekannt, durch Tausch oder Kauf an das Kloster Geringswalde gekommen. ${ }^{24}$ Somit wird man mit Sicherheit den Pfarrsprengel Schönerstädt den Schönburgischen Besitzungen zurechnen können. Die beiden Dörfer Ascholveshagen (Aschershain) und Vleminge (Flemmingen), gelegen im Pfarrsprengel Hartha, erhielt das Kloster 1288 von Friedrich von Schönburg im Zusammenhang mit der Bestätigung aller anderen Klosterbesitzungen. ${ }^{25}$

Man kann also davon ausgehen, dass die Orte des Pfarrsprengels Hartha ebenfalls von den Schönburgern angelegt worden sind. Jedenfalls war er im 14. Jahrhundert im Besitz von Veit von Schönburg. Dies geht aus der Leibgedingeverschreibung für Ilse, Gemahlin des Dietrich von Beerwalde auf Kriebstein, durch Markgraf Wilhelm (1343-1407) 1404 hervor, ${ }^{26}$ in der es heißt: das Städtchen Hartha mit dem Kirchlehen, Arras, den Zoll zu Flemmingen, Stein, Richzenhain, Dietenhain und Saalbach, die Dietrich von Beerwalde von dem „edeln ern

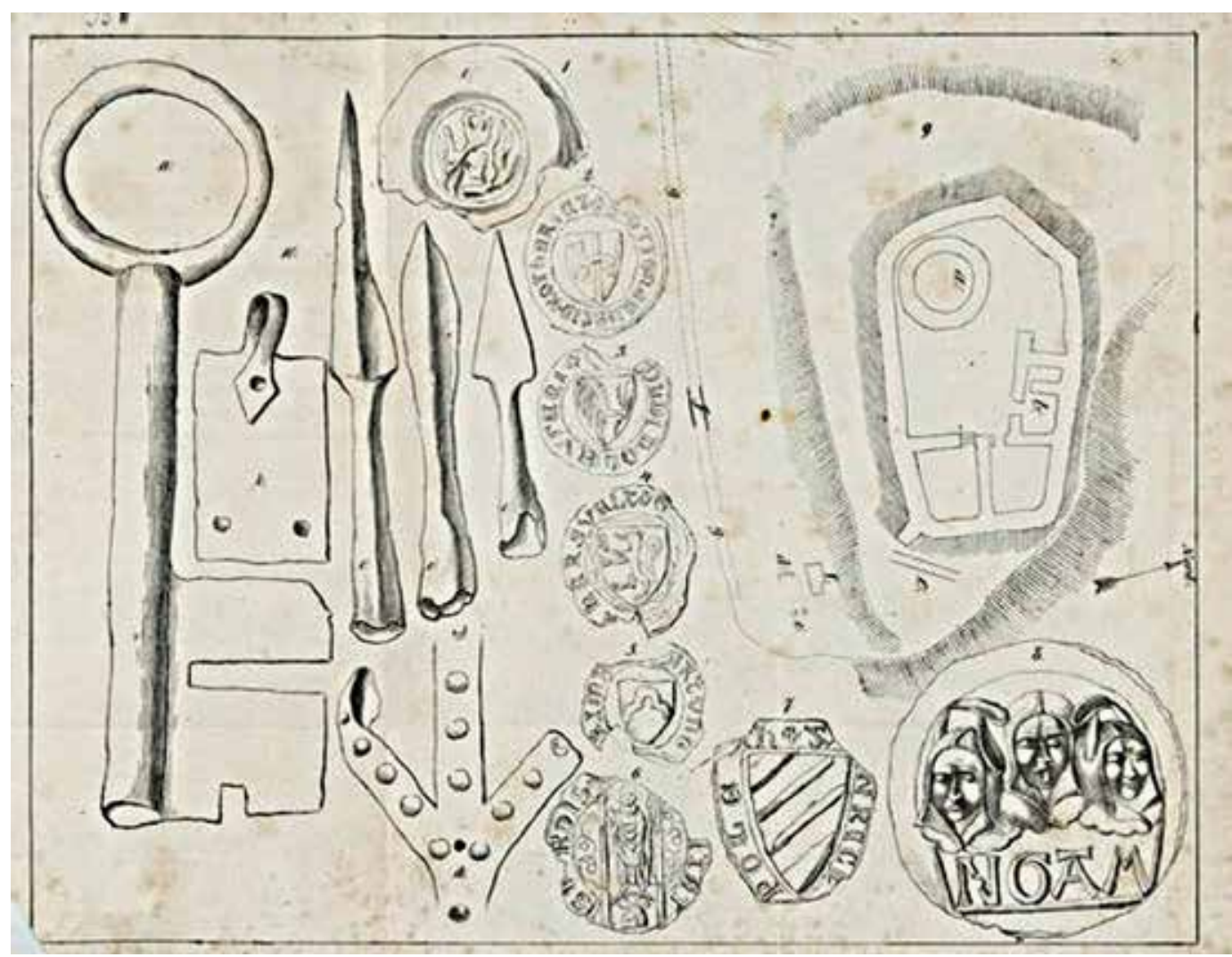

12 Müller übersetzt „fundum castri destructi et opidi desolati“ mit "Grund und Boden der zerstörten Burg und verwüsteten Ortschaft".

13 Neef, Ernst: Die natürräumliche Gliederung Sachsens (6. Teil). In: Sächsische Heimatblätter 6 (1960), S. 565579, hier S. 565-572, $578 \mathrm{f}$.

14 Billig, Gerhard: Burgenarchäologische und siedlungskundliche Betrachtungen zum Flußgebiet der Zschopau und der Freiberger Mulde. In: Zeitschrift für Archäologie 15 (1981), S. 265-297, hier S.265f.

15 Posse, Otto (Hrsg.): Codex diplomaticus Saxoniae regiae. Erster Haupttheil, Abteilung A, Bd. 2. Urkunden der Markgrafen von Meißen und Landgrafen von Thüringen 11001195. Leipzig 1889, S. 189 f., Nr. 277; Schlesinger, Walter: Die Landesherrschaft der Herren von Schönburg, Münster/Köln 1954, S. 18; Kobuch, Manfred: Leisnig im Hochmittelalter. In: Burgenforschung aus Sachsen 8 (1996), S. 11-32, hier S. $18 \mathrm{f}$.

16 Schwabenicky, Wolfgang: Sachsenburg im Mittelalter. Mittweida 1997, S. $16 \mathrm{f}$.

17 Bosl, Karl: Die Reichsministerialen der Salier und Staufer. Teil 2. Stuttgart 1951, S. 510 f.; Schlesinger, Walter: Die Landesherrschaft der Herren von Schönburg, Münster/Köln 1954 S. 28-31.

18 Graber, Tom (Hrsg.): Codex Diplomaticus Saxoniae. Zweiter Hauptteil. Bd. 19. Urkundenbuch des Zisterzienserklosters Altzelle, Erster Teil 11621249. Hannover 2006, S. 12-14.

19 Schön, Theodor: Geschichte des Fürstlichen und Gräflichen Gesammthauses Schönburg. Band I des Urkundenbuches der Herren von Schönburg. Waldenburg 1901 S. 14, Nr. 24; Schön, Theodor: Geschichte des Fürstlichen und Gräflichen Gesammthauses Schönburg. Nachträge zu Band I-VIII des Urkundenbuches der Herren von Schönburg. Waldenburg 1910, S. 16, Nr. 29.

20 Sächsisches Staatsarchiv, Staatsarchiv Leipzig, 20438 Rittergut Klostergeringswalde, Nr. 107, Erbregister über das Closter und Städtlein Göringswalda... 1590 (Abschrift von 1788). Das Erbzinsregister ist anlässlich des Verkaufs der Schönburgischen Besitzes um Geringswalde 1590 an Kurfürst Christian I. erstellt worden. Vgl. Schön, Theodor: Geschichte des Fürstlichen und Gräflichen Gesammthauses Schönburg. Band VIII des Urkundenbuches der Herren von Schönburg, Zweiter Teil. Waldenburg 1906, S. 109117, Nr. 242.

21 Bernhardi 1777 (wie Anm. 2), S. 55 f.; Tobias 1865 (wie Anm. 5), S. 17; Hofmann/Müller 1920 (wie Anm. 6) S. 58,70 .

Geringswalde, Altes Schloss, Funde und Plan aus dem 18. Jahrhundert nach Gautsch 1843 
Schönburgische Besitzungen im 13. Jahrhundert Zeichnung: Wolfgang Schwabenicky

22 Battré, Herta: Beiträge zur Geschichte des Klosters Buch. Diss. Leipzig 1951, S. 18; Schöttgen, Christian/Kreysig, Georg Christoph: Diplomataria et sriptores historiae Germanicae medii aevi. Bd. 2. Altenburg 1755, S. 184 f., Nr. XXXIV

23 Schlesinger, Walter: Die Schönburgischen Lande bis zum Ausgang des Mittelalters. Dresden [1935], S. 15.

24 Sieber, J. G. 1929 (wie Anm. 9), S. 76

25 Tobias 186 5(wie Anm. 5), S. 25 f.; Schön 1901 (wie Anm. 19), S. 30 f., Nr. 85; Schön 1910 (wie Anm. 19), S. 31 f., Nr. 4

26 Sieber, J. G.: Beiträge zur Geschichte der Stadt Hartha. In: Hermann Gläsers Harthaer Heimatbuch. Bd. 2. Hartha 1930, S. 1-120, hier S. 17. Markgraf Wilhelm hatte sich zunächst die Gerichtsbarkeit über die von Dietrich von Beerwalde erworbenen Dörfer vorbehalten. Er verpfändete die Gerichte 1405 an Dietrich, dessen Frau Ilse, Tochter Clara und den Neffen Conrad, Dietrich, Balthasar, Caspar und Melchior, vgl. Hubert Ermisch (Hrsg.): Codex diplomaticus Saxoniae regiae. Erster Haupttheil, Abteilung B, Bd. 2. Urkunden der Markgrafen von Meißen und Landgrafen von Thüringen 1396-1406. Leipzig 1902, S. 451, Nr. 650.

27 Hubert Ermisch (Hrsg.): Codex diplomaticus Saxoniae regiae. Erster Haupttheil, Abteilung B, Bd. 1. Urkunden der Markgrafen von Meißen und Landgrafen von Thüringen 13811395. Leipzig 1899, S. 88, Anm. zu Nr. 125; Sieber 1930 (wie Anm. 26), S. 14.

28 CDS B I (wie Anm. 27), S. 244-246, Nr. 315

29 Bei dem „Gerunkswald“ handelt es sich um das Waldgebiet nordwestlich von Geringswalde. Die zwei Teile des Waldes werden durch den Harthaubach getrennt. Für den nördlichen Teil kam später der Name „Schönburger Wald“, für den südlichen Teil der Name „Fürstenwald“ auf.

30 CDS I B I (wie Anm. 27), S. 256-258, Nr. 332

31 Billig 1981 (wie Anm. 14), S. 277.

2 Kretzschmar, Johannes: Münzdatierte frühmittelalterliche Gefäße aus dem nordwestlichen Sachsen und ihre Bedeutung. In: Sachsens Vorzeit 31939 (1940), S. 89-96, hier S. 96; Stoll, Hans-Joachim: Münzschatzgefäße. Weimar 1985, S. 18 und Tafel XI.

3 Sieber 1929 (wie Anm.9), S. 64.

34 Walther, Hans: Die Orts- und Flurnamen des Kreises Rochlitz. Halle 1957, S. 22 (4. Arras)

35 Kötzschke, Rudolf: Ländliche Siedlungen und Agrarwesen in Sachsen. Remagen 1953, S. 164 f.; Walther 1957 (wie Anm. 34), S. $267 \mathrm{f}$.

36 Walther 1957 (wie Anm. 34), S. 266.

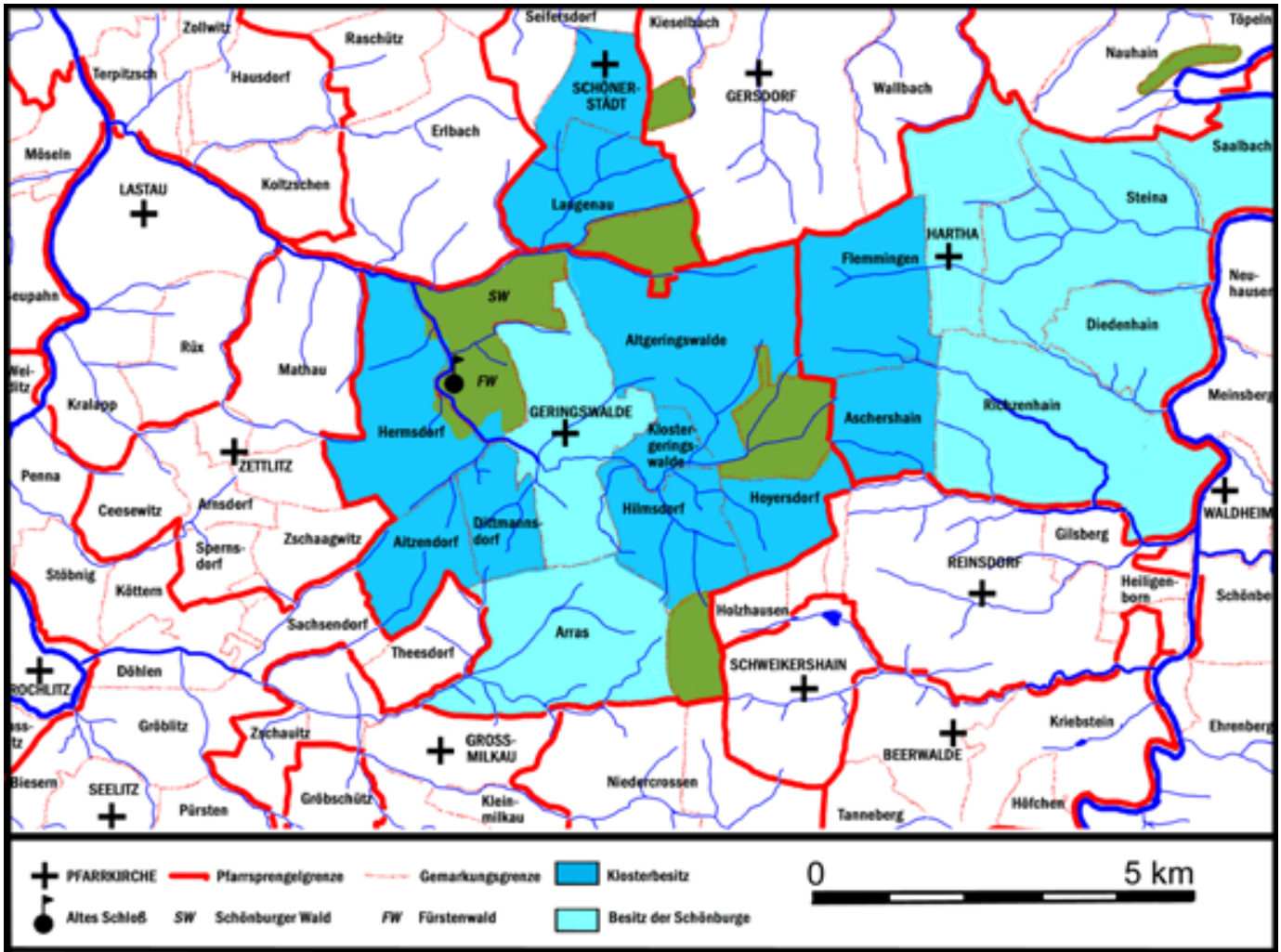

Vite von Schonburg, hern zcu Gluchaw“ gekauft hat. Der Kauf hat nach 1395 stattgefunden, denn in der Leibgedingeverschreibung für Ilse vom 26. Januar dieses Jahres ist nur Waldheim mit den zugehörigen Dörfern, nicht aber Hartha genannt. ${ }^{27}$

Wie es scheint, hing der Verkauf der noch in seinem Besitz befindlichen Dörfer des Geringswalder Gebietes (ohne das Kloster) mit dem Ausgang der Streitigkeiten zwischen Markgraf Wilhelm I. und Veit I. von Schönburg zusammen. Auf Veranlassung König Wenzels (1361-1419) wurden durch Friedrich, Pfalzgraf bei Rhein und Herzog in Bayern (um 1339-1393), und Markgraf Jost von Mähren (1351-1411) im Dezember 1389 auf der Burg Betlern (Žebrák) in Böhmen diese Auseinandersetzungen durch einen Schiedsspruch beendet. ${ }^{28}$ Für unser Gebiet wichtig sind folgende Festlegungen: Der „Gerunkswald“ wurde geteilt, wobei die eine Hälfte der Markgraf erhielt, die andere im Eigentum von Veit von Schönburg blieb. ${ }^{29}$ Das weitere Ergebnis war ein in Rochlitz am 20. April 1390 ausgestelltes Diktat $^{30}$, nach dem sich Veit von Schönberg verpflichtete, dem Markgrafen Wilhelm mit seinen Schlössern Glauchau und Waldenburg zu dienen, mit dem Kloster Geringswalde keinen Streit mehr zu haben und ihm alles zurückzugeben, sowie andere Verpflichtungen, die in unserem Zusammenhang nicht von Bedeutung sind.
Die Schönburgischen Besitzungen um Geringswalde waren ein kleines aber geschlossenes Gebiet, das im Zuge der bäuerlichen Kolonisation des Erzgebirgsvorlandes entstanden ist. Die Besiedlung erfolgte wahrscheinlich von Osten nach Westen, wobei von hier aus das Gebiet am besten zu erreichen war, da hier der von Leisnig über Waldheim führende Böhmische Steig die Zschopau querte. ${ }^{31}$ Die Herkunft der Siedler ist unklar. Man hat bisher angenommen, dass die Schönburge hier Flamen angesetzt haben. Anlass war dafür vor allem der Ortsname von Flemmingen. Gestützt wird diese Annahme auch durch den Fund eines mit 15 Münzen gefüllten Kugeltopfes ${ }^{32}$, der nördlicher Herkunft sein kann. Der Topf wurde in der ersten Hälfte des 19. Jahrhunderts entdeckt. Die genauen Fundumstände sind nicht bekannt. Eine flämische Gründung soll auch Arras sein. ${ }^{33}$ Dem entgegen steht aber hier wie auch den anderen Dörfern dem Maße nach die Verwendung der fränkischen Hufe von 49 Ackern (= 27,1 Hektar). ${ }^{34}$ Die Herkunft der Siedler aus den Hufengrößen zu ermitteln, ist ohnehin problematisch. ${ }^{35}$ Bei Geringswalde, Aitzendorf, Arras und Flemmingen kann es sich auch um Ortsnamensübertragungen aus westlicheren Gebieten handeln. ${ }^{36}$ Auffällig ist bei den Dörfern um Hartha und Geringswalde, dass es sich nicht um klassische Waldhufendörfer handelt, sondern um Dörfer mit einer sogenannten Breitstreifenflur, wie dies bereits von Walter Schlesinger he- 


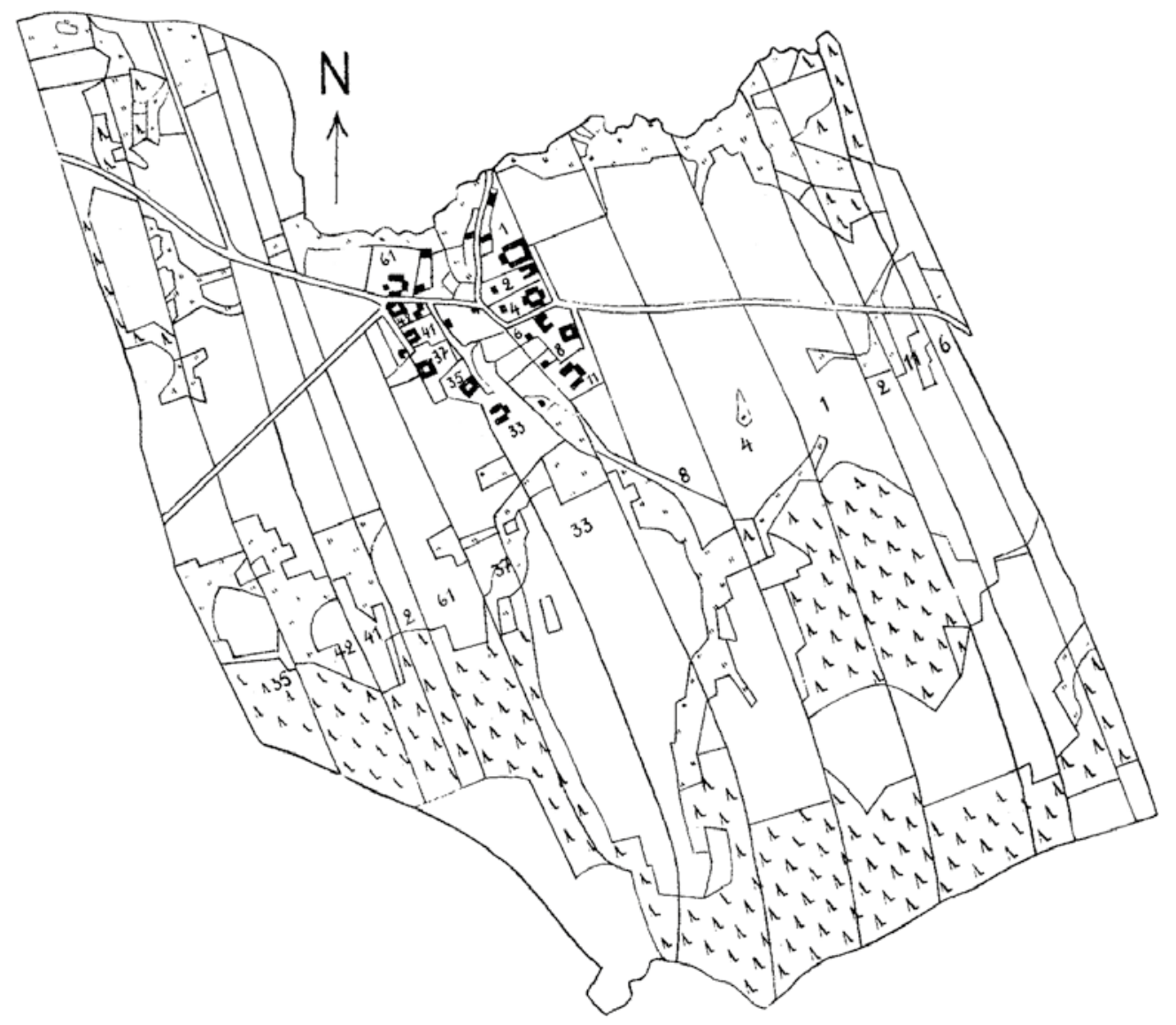

rausgestellt wurde. ${ }^{37}$ Diese Breitstreifenfluren setzten sich aus waldhufenähnlichen Streifen zusammen, die geradlinig über die ganze Flur liefen, wobei als Ortsform noch das geschlossene Angerdorf vorkommt, aber auch schon die Gehöftreihe. ${ }^{38}$ Die Breitstreifenflur wird als eine ältere Flurform angesehen, die in hiesiger

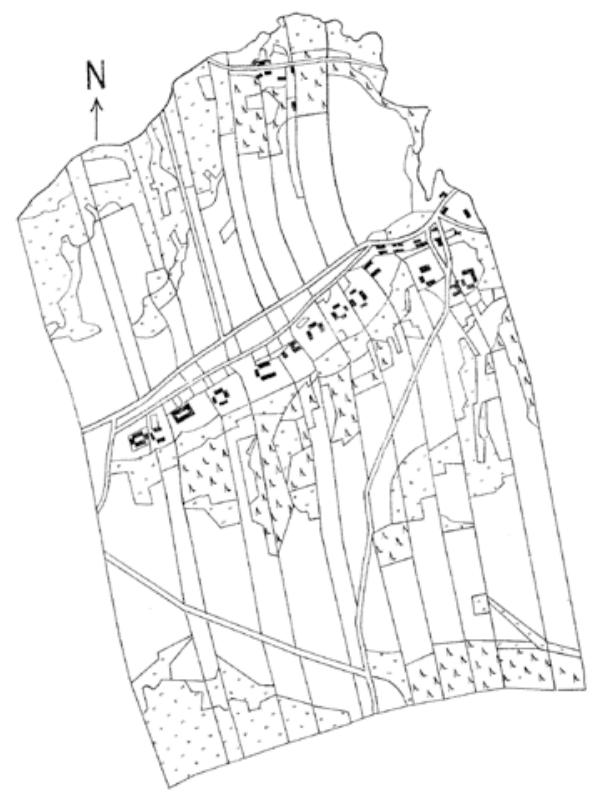

Gegend bereits in der ersten Hälfte des 12. Jahrhunderts angewandt wurde.

Die Geringswalder Herrschaft der Schönburger bestand also aus den Pfarrsprengeln Geringswalde, Hartha und Schönerstädt, wobei letzeres möglicherweise nicht von Anfang an dazu gehört hat. In diesem Gebiet liegt eine bedeutende Burgstätte, nämlich das Alte Schloss im Fürstenwald. Es handelt sich um eine mehrkernige Anlage auf einem Bergsporn von insgesamt 190 Meter Länge und 50 Meter Breite. Die Burgkerne sind jeweils von Gräben getrennt. An der Nordseite befindet sich ein durchlaufender Graben, der die Spornspitze umzieht. ${ }^{39}$ Auf der Spornspitze befinden sich noch geringe Reste der im 18. Jahrhundert ausgegrabenen Burgruine. Bei den Ausgrabungen wären romanische Bauteile gefunden worden, wonach die Burg von Richard Steche um 1200 datiert wird. ${ }^{40}$ Datierende Funde sind heute nicht mehr vorhanden. ${ }^{41}$ Man wird allerdings die Bauzeit der Burg um 1200 als richtig annehmen können, wenn man dazu die Entstehungszeiten anderer Burgen in dem im 12. Jahrhundert besiedelten Räumen im Erzgebirgsvorland und Erzgebirge vergleicht, wie sie in den letzten Jahrzehnten präzisiert werden konnten. Die steinerne Burg Lichtenwalde entstand nach den Grabungser-
Hilmsdorf, Flurplan um 1840 Zeichnung nach Kötzschke 1957 (wie Anm. 35), Abb. 25
37 Schlesinger, Walter: Die Besied- lung des Landes um Rochlitz. In Bernstein, Albert (Hrsg.): Das Buch der Landschaft Rochlitz. Rochlitz 1936, S. 102-115, hier S. 111.
38 Langer, Johannes: Heimatkundliche Streifzüge durch Fluren und Orte des Erzgebirges und seines Vorlandes. Schwarzenberg 1931, S. 21 f.; Krü- ger, Rainer: Typologie des Waldhu- fendorfes nach Einzelformen und de- ren Verbreitungsmustern. Göttingen 1967, S. $45 \mathrm{f}$.
39 Geupel, Volkmar: Die geschützten Bo- dendenkmale im Bezirk Karl-Marx- Stadt. Dresden 1983, S. 64 f.
40 Steche, Richard: Beschreibende Dar- stellung der älteren Bau- und Kunst- denkmäler des Königreichs Sach- sen. 14. Heft: Amtshauptmannschaft Rochlitz. Dresden 1890, S. 10-12.
41 In der Sammlung Pfau (Museum Rochlitz) befindet sich eine Reihe Lesefunde von mittelalterlicher Ke- ramik mit dem Fundort Geringswal- de, ohne dass sie dem Alten Schloß eindeutig zugeordnet werden kön- nen (Landesamt für Archäologie Sachsen, Ortsakte Geringswalde).

Dittmannsdorf, Flurplan um 1840 Zeichnung nach Kötzschke 1957 (wie Anm. 35), Abb. 29 
Flemmingen, Flurplan um 1840 Zeichnung nach Kötzschke 1957 (wie Anm. 35), Abb. 32

42 Degenkolb, Peter/Geupel, Volkmar: Die Burgen Lichtenwalde und Zschopau im Licht neuer Forschungsergebnisse. In: Im Dienste der historischen Landeskunde. Festgabe für Gerhard Billig zum 75. Geburtstag. Beucha 2002, S. 151-166, hier S. 155.

43 Geupel, Volkmar/Hoffmann, Yves: Die Ausgrabung der Burg Schellenberg. In: Schellenberg-Augustusburg, Beiträge zur 800-jährigen Geschichte. Augustusburg 2006, S. 161-199, hier S. 181.

44 Schwabenicky, Wolfgang: Schloß Sachsenburg, Geschichte und Baugeschichte aufgrund archivalischer und bauarchäologischer Untersuchungen. Sachsenburg 2009, S. 9, 39,104

45 Schwabenicky, Wolfgang: Bergstädte des 12. bis 14. Jahrhundert in Sachsen. In: Hoffmann, Yves/Richter, Uwe (Hrsg.): Die Frühgeschichte Freibergs im überregionalen Vergleich. Städtische Frühgeschichte - Bergbau - früher Hausbau. Halle 2013, S. 211-224, hier S. 213-215.

46 Sieber 1930 (wie Anm. 26), S. 20.

47 Schön 1906 (wie Anm. 19), S. 112

48 Sächsisches Staatsarchiv, Staatsarchiv Leipzig, 20438 Rittergut Klostergeringswalde, Nr. 107 (wie Anm. 20), Bl. 87 und $87 \mathrm{~b}$

49 Hofmann/Müller 1920 (wie Anm. 6), S. 136; Schlesinger 1935 (wie Anm. 23), S. 15

50 Schlesinger 1935 (wie Anm. 23), S. 93-95; Billig, Gerhard: Das Reich und die Vögte. Plauen 2002, S. 79-82.

51 Posse 1914 (wie Anm. 10), S. 9.

Autor

Dr. phil.

Wolfgang Schwabenicky Altmittweida

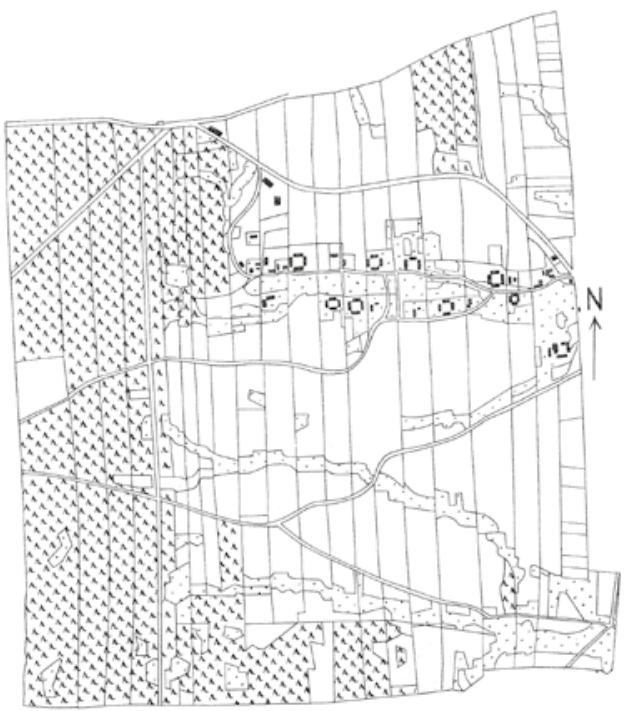

gebnissen nach 1200 , etwa um 1220 bis $1230 .{ }^{42}$ Eine ähnliche Datierung für die Burg Schellenberg (Augustusburg) erbrachten auch die dortigen archäologischen Untersuchungen. ${ }^{43}$ Die Anfänge der Sachsenburg bei Frankenberg liegen nach dem Baubefund und der ältesten ergrabenen Keramik (vorgraublaue Ware) um $1200 .{ }^{44}$

Unterhalb des Alten Schlosses fast auf dem Talboden befand sich eine Mühle, deren Aufschlaggraben und Standort des Mühlrades heute noch gut im Gelände erkennbar sind. Eine Mühle gehörte zu jeder Burg, da der Erbzins immer in Getreide geliefert wurde, die Weiterverarbeitung zur Eigenversorgung aber in den Händen der Zinsberechtigten selbst lag.

Etwa gleichzeitig mit der Burg im Fürstenwald wird auch die Stadt Geringswalde durch die Schönburger als Nahhandelsort für die umgebenden Dörfer gegründet worden sein. Der tägliche Markt war das, was für die Menschen des späten Mittelalters den Begriff Stadt (oder auch Weichbild) für einen Ort rechtfertigte und auch den Anfang einer Stadt ausmachte. Das heißt die ständige Anwesenheit von Händlern, zu denen auch die Handwerker zählten, da sie letztendlich auch kauften und nach der Bearbeitung des Eingekauften ihre Produkte wieder verkauften. Der freie Markt, das heißt der regelmäßig einmal an einem bestimmten Tag in der Woche stattfindende Markt (Wochenmarkt), kam dann erst zur Stadt dazu. ${ }^{45}$ Nach der Besiedlung der ehemaligen Waldgebiete kam es auch zu einer Neutrassierung der Verkehrswege. Die Böhmischen Steige verloren an Bedeutung. Sie wurden nur noch teilweise in die neu entstehenden Straßen einbezogen. Im Raum Geringswalde entstand eine neue Straße, die von Schönerstädt über Langenau nach Geringswalde führte und weiter über Altgeringswalde, Flemmingen nach Hartha, schließlich über Richzenhain nach Waldheim. Die Bedeutung dieser Straße erhellt aus dem Vorhandensein zweier Zollstätten. Einmal ist das der Zoll zu Flemmingen auf Harthaer Flur. ${ }^{46}$ Zum anderen ist das der Zoll zu Schönerstädt. Er wird im Kaufvertrag mit Kurfürst Christian I. von Sachsen (1560-1591) genannt; er brachte jährlich etwa 15 Gulden an Einnahmen. ${ }^{47}$ Das Geld hatte der Richter von Schönerstädt einzunehmen, der dafür keine Frondienste zu leisten hatte. ${ }^{48}$ Die starke Frequentierung der Straße wird auch deutlich an dem gewaltigen Bündel von Hohlwegen am Anstieg der Straße nach Geringswalde südlich des unteren Ortsausganges von Langenau. Der Verkehr auf der Straße war nicht die Ursache für die Gründung der Stadt, wirkte sich aber positiv auf ihre Entfaltung aus. Später war Geringswalde eine Exportgewerbestadt auf der Basis des Textilhandwerks. Sie gelangte aber nicht zur Ratsverfassung, sondern das Regiment wurde immer von einem Richter mit Schöppen geführt.

Die Zerstörung der Burg und der Stadt Geringswalde soll zwischen 1212 und 1214 durch die Böhmen erfolgt sein. ${ }^{49}$ Für die Wiedererrichtung der Burg gab es keine ausreichende Veranlassung, da der Hauptsitz der Schönburger bereits seit längerem im Gebiet von Glauchau lag. ${ }^{50}$ Einen Nahmarkt brauchte man bei Geringswalde weiterhin, so dass die Stadt wieder aufgebaut wurde. Die Annahme, dass dies an anderer Stelle geschah, ist unbegründet. Die Lage der Stadt auf dem Höhenzug zwischen Klosterbach und Altgeringswalder Bach ist die günstigste. Der Begriff „fundus castri“ wurde immer mit „Grund und Boden der Burg“ übersetzt und damit den Standort der Burg gemeint. Nun wurde von Otto Posse schon erwogen, dass die Urkunde von den Nonnen geschrieben wurde, die Schwierigkeiten mit dem Latein hatten. ${ }^{51}$ Wenn man „V areas et fundum castri destructi et opidi desolati“ zusammenfast zu „fünf Grundstücken auf dem Grund und Boden der zerstörten Burg und verlassenen Stadt“, so ist das eine Einschränkung. Das Kloster erhielt nämlich nicht alle Liegenschaften der Burg und der Stadt, sondern nur fünf. Vor diesem Passus werden „XVI mansos in antiquo gerungswalde“ (16 Hufen in Altgeringswalde) aufgeführt; das ist das ganze Dorf. Nach dem Erbzinsregister von 1590 hat Altgeringswalde 17 Hufen. Bei den fünf (städtischen) Grundstücken handelt es sich um die Flur von Klostergeringswalde, auf der das Kloster errichtet worden ist. Es gibt demzufolge keinen Grund, hier als Vorgängerbebauung eine Burg zu suchen. 